\title{
Loss of $\mathrm{ncm}^{5}$ and $\mathrm{mcm}^{5}$ wobble uridine side chains results in an altered metabolic profile
}

\author{
Tony Karlsborn $^{1}$ • A. K. M. Firoj Mahmud ${ }^{1} \cdot$ Hasan Tükenmez $^{1} \cdot$ Anders S. Byström $^{1}$ (D)
}

Received: 8 July 2016/Accepted: 14 September 2016/Published online: 27 September 2016

(c) The Author(s) 2016. This article is published with open access at Springerlink.com

\begin{abstract}
Introduction The Elongator complex, comprising six subunits (Elp1p-Elp6p), is required for formation of 5-carbamoylmethyl $\left(\mathrm{ncm}^{5}\right)$ and 5-methoxycarbonylmethyl $\left(\mathrm{mcm}^{5}\right)$ side chains on wobble uridines in 11 out of 42 tRNA species in Saccharomyces cerevisiae. Loss of these side chains reduces the efficiency of tRNA decoding during translation, resulting in pleiotropic phenotypes. Overexpression of hypomodified $\mathrm{tRNA}_{\mathrm{s}^{2} \mathrm{UUU}}^{\mathrm{Lyy}}, \mathrm{tRNA}_{\mathrm{s}^{2} \mathrm{UUG}}^{\mathrm{Gln}}$ and tRNA $_{\mathrm{s}^{2} U U C}^{\mathrm{Glu}}$, which in wild-type strains are modified with $\mathrm{mcm}^{5} \mathrm{~s}^{2} \mathrm{U}$, partially suppress phenotypes of an elp3 $\Delta$ strain. Objectives To identify metabolic alterations in an elp3A strain and elucidate whether these metabolic alterations are suppressed by overexpression of hypomodified tRNA $\mathrm{s}^{2} \mathrm{UUU}$, $\mathrm{tRNA}_{\mathrm{s}^{2} \mathrm{UUG}}^{\mathrm{Gln}}$ and tRNA $\mathrm{s}^{2} \mathrm{GUC}$.

Method Metabolic profiles were obtained using untargeted GC-TOF-MS of a temperature-sensitive elp3 $\Delta$ strain carrying either an empty low-copy vector, an empty high-copy vector, a low-copy vector harboring the wild-type ELP3 gene, or a high-copy vector overexpressing $\operatorname{tRNA}_{\mathrm{s}^{2} \mathrm{UUU}}^{\mathrm{Lys}}, \mathrm{tRNA}_{\mathrm{s}^{2} \mathrm{UUG}}^{\mathrm{Gln}}$ and tRNA $\mathrm{s}^{2} \mathrm{GUC}$. The temperature sensitive elp3A strain derivatives were cultivated at
\end{abstract}

A. K. M. Firoj Mahmud and Hasan Tükenmez has contributed equally to this work.

Electronic supplementary material The online version of this article (doi:10.1007/s11306-016-1120-8) contains supplementary material, which is available to authorized users.

Anders S. Byström

Anders.Bystrom@molbiol.umu.se

1 Department of Molecular Biology, Umeå University, 90187 Umeå, Sweden permissive $\left(30^{\circ} \mathrm{C}\right)$ or semi-permissive $\left(34^{\circ} \mathrm{C}\right)$ growth conditions.

Results Culturing an elp3 $\Delta$ strain at 30 or $34{ }^{\circ} \mathrm{C}$ resulted in altered metabolism of 36 and $46 \%$, respectively, of all metabolites detected when compared to an elp3 $\Delta$ strain carrying the wild-type ELP3 gene. Overexpression of hypomodified $\quad \operatorname{tRNA}_{\mathrm{s}^{2} \mathrm{UUU}}^{\mathrm{Lys}}, \mathrm{tRNA}_{\mathrm{s}^{2} \mathrm{UUG}}^{\mathrm{Gln}}$ and tRNA $\mathrm{s}^{2} \mathrm{UUC}$ suppressed a subset of the metabolic alterations observed in the elp3 $\Delta$ strain.

Conclusion Our results suggest that the presence of $\mathrm{ncm}^{5}$ and $\mathrm{mcm}^{5}$-side chains on wobble uridines in tRNA are important for metabolic homeostasis.

Keywords Elongator complex - tRNA wobble uridine modifications - Translation - ELP3 - Metabolomics · Metabolic profiling

\section{Introduction}

In eukaryotes, the Elongator complex is highly-conserved and comprises six subunits (Elp1p-Elp6p) (Otero et al. 1999; Y. Li et al. 2001; Krogan and Greenblatt 2001; Winkler et al. 2001; Hawkes et al. 2002; Nelissen et al. 2005). The complex is required for formation of 5-carbamoylmethyluridine $\left(\mathrm{ncm}^{5} \mathrm{U}\right), 5$-methoxycarbonylmethyluridine $\left(\mathrm{mcm}^{5} \mathrm{U}\right)$ and 5-methoxycarbonylmethyl-2thiouridine $\left(\mathrm{mcm}^{5} \mathrm{~s}^{2} \mathrm{U}\right)$ modifications at wobble positions in tRNAs (Huang et al. 2005; Esberg et al. 2006; Chen et al. 2009; Lin et al. 2013; Mehlgarten et al. 2010; Karlsborn et al. 2014a). In Saccharomyces cerevisiae, loss-of-function mutations in any gene encoding an Elongator complex subunit gives rise to a multitude of phenotypes linked to several different cellular processes (Otero et al. 1999; Wittschieben et al. 1999; Winkler et al. 2002; Rahl et al. 
2005; Tigano et al. 2015; Nedialkova and Leidel 2015; Frohloff et al. 2001; Chen et al. 2011; Q. Li et al. 2009). Phenotypes observed in yeast Elongator mutants, except the tRNA modification defect, are suppressed by overexpression of tRNA $\mathrm{s}^{2} \mathrm{LUU}$ and tRNA $\mathrm{s}^{2} \mathrm{GUG}$, which have the $\mathrm{mcm}^{5}$ $\mathrm{s}^{2} \mathrm{U}$ modification in wild-type yeast. This discovery highlighted the importance of Elongator-complex-dependent tRNA modifications in translation (Esberg et al. 2006).

In an elp $3 \Delta$ mutant enrichment of lysine-AAA codons in mRNAs decoded by tRNA ${ }_{\mathrm{mcm}^{5} \mathrm{~s}^{2} \mathrm{UUU}}^{\mathrm{Lys}}$ having the Elongator complex dependent wobble uridine modification $\mathrm{mcm}^{5} \mathrm{~s}^{2} \mathrm{U}$ result in reduced protein expression (Bauer et al. 2012). Replacing these lysine-AAA codons with the near-cognate G-ending AAG codon, decoded by a tRNA isoacceptor not requiring the Elongator complex dependent wobble uridine modification improved protein expression from the codon altered gene (Fernandez-Vazquez et al. 2013; Bauer et al. 2012). Moreover, ribosomal profiling studies performed with Elongator mutants revealed ribosomal pausing at the lysine-AAA and glutamine-CAA codons (Nedialkova and Leidel 2015; Zinshteyn and Gilbert 2013) and possibly the glutamic acid-GAA codons (Zinshteyn and Gilbert 2013). These results support the previous suggestion that the presence of the $\mathrm{mcm}^{5} \mathrm{~s}^{2} \mathrm{U}$ modification in $\mathrm{tRNA}_{\mathrm{mcm}^{5} \mathrm{~s}^{2} \mathrm{LUU}}^{\mathrm{Lys}}$ and tRNA $\mathrm{mcm}_{\mathrm{m} \mathrm{s}^{2} \mathrm{UUG}}^{\mathrm{Gln}}$ enhance translational efficiency (Esberg et al. 2006), probably because of improved codon-anticodon interactions (Johansson et al. 2008; Durant et al. 2005; Bauer et al. 2012; Vendeix et al. 2012; Rezgui et al. 2013; Tükenmez et al. 2015). However, whether the phenotypes are caused by global reduction of protein expression or altered protein expression from specific mRNAs, leading to downstream effects, is unknown. Moreover, whether the loss of modified wobble uridines causes metabolic alterations is yet to be determined.

Our study demonstrates that a large number of metabolites within an elp3 $\Delta$ strain undergoes perturbed metabolism. Furthermore, the range of metabolites with altered levels expanded with growth of the elp $3 \Delta$ strain at $34{ }^{\circ} \mathrm{C}$; this is probably an effect of the temperature sensitivity phenotype of the elp $3 \Delta$ mutant. Our study also shows that elevated levels of $\mathrm{tRNA}_{\mathrm{s}^{2} \mathrm{UUU}}^{\mathrm{Lys}}, \mathrm{tRNA}_{\mathrm{s}^{2} \mathrm{UUG}}^{\mathrm{Gln}}$ and $\mathrm{tRNA}_{\mathrm{s}^{2} \mathrm{UUC}}^{\mathrm{Glu}}$ in the elp3 3 strain suppress some, but not all metabolic alterations.

\section{Methods}

\subsection{Yeast strains, media, and genetic procedures}

Yeast strains and plasmids used in this study are found in Online Resource 1 (Christianson et al. 1992; Lu et al. 2005; Sikorski and Hieter 1989). Genetic procedures, media, and yeast transformation have been described previously (Burke et al. 2000; Gietz and Schiestl 2007). An elp3 null mutant was generated by linear transformation of the diploid strain UMY2016/UMY2026 with an elp3::kanMX4 fragment (fragment amplified by polymerase chain reaction (PCR)) generated from the elp $3 \Delta$ strain in the yeast deletion collection (Open Biosystems). Transformants were selected on YEPD plates containing $200 \mu \mathrm{g} / \mathrm{ml}$ of G418. The heterozygous diploid generated was sporulated and tetrad dissection generated haploids UMY4238 and UMY4239. Insertion of the elp3::KanMX4 cassette in UMY4239 was verified by PCR and high performance liquid chromatography (HPLC) analysis was used to determine the status of the wobble uridine nucleosides: $\mathrm{ncm}^{5} \mathrm{U}, \mathrm{mcm}^{5} \mathrm{U}$ and $\mathrm{mcm}^{5} \mathrm{~s}^{2} \mathrm{U}$ in yeast tRNA (Huang et al. 2005).

\subsection{Cell sampling and metabolite extraction using untargeted GC-TOF-MS metabolomics}

Strains UMY4239 (elp3::KanMX4) and UMY4238 were transformed with one of the following plasmids: empty pRS315, pRS315 containing the wild-type ELP3 gene, pRS425 or pRS425 containing the tRNA genes $t K(U U U)$, $t Q(U U G)$ and $t E(U U C)$. Three replicates of each strain derivative were cultivated in synthetic defined media at either 30 or $34{ }^{\circ} \mathrm{C}$ until cell density was $\sim 0.5 \mathrm{OD}_{600}$ units. At $\sim 0.5 \mathrm{OD}_{600}$ units, cells amounting to $1 \mathrm{OD}$ unit were harvested in triplicate from each biological replicate by centrifugation at $0{ }^{\circ} \mathrm{C}$. The supernatant was discarded and the cells were washed with $2 \mathrm{ml}$ of ice-cold phosphate buffered saline (PBS) then centrifuged again at $0{ }^{\circ} \mathrm{C}$. The supernatant was discarded and pellets were suspended in a 90:10 mixture of Methanol and MilliQ (MQ) water which was pre-chilled on dry ice. Suspended pellets were stored at $-80{ }^{\circ} \mathrm{C}$ until metabolite extraction.

Metabolites were extracted by grinding the pellets with glass beads for $3 \mathrm{~min}$ at $30 \mathrm{~Hz}$ followed by centrifugation at $14,000 \mathrm{RPM}$ for $10 \mathrm{~min}$. A $200 \mu \mathrm{L}$ aliquot of the supernatant was transferred to a GC-vial and evaporated using a SpeedVac. Derivatization of the metabolic extract was performed using $30 \mu \mathrm{L}$ of methoxyamine (16 h at room temperature). The extract was then trimethylsilylated by adding $30 \mu \mathrm{L}$ of $N$-methyl- $N$-trimethylsilyltrifluoroacetamide (MSTFA) to the vial and incubating for $1 \mathrm{~h}$ at $25^{\circ} \mathrm{C}$. Subsequently, $30 \mu \mathrm{L}$ of heptane containing $15 \mathrm{ng} /$ $\mu \mathrm{L}$ methyl stearate was added to the vial.

Samples were analysed using combined gas chromatography with time-of-flight mass spectrometry (GC/ TOFMS). For retention indices, an $n$-alkane series (C8C40) was included in the analysis (Schauer et al. 2005). A $1 \mu \mathrm{L}$ volume of derivatized sample was injected into a split/splitless injector, in splitless mode, on an Agilent CTC PAL Systems Autosampler with a $10 \mu \mathrm{L}$ syringe (Agilent 
Technologies, Atlanta, GA, USA). The autosampler injected samples into an Agilent Technologies 7890A GC System (Agilent Technologies, Atlanta, GA, USA). The Agilent Technologies 7890A GC System was equipped with a $30 \mathrm{~m} \times 0.250 \mathrm{~mm}$-diameter fused, silica capillary column with a bonded $0.25 \mu \mathrm{m}$ Durabond DB-5MSUI stationary phase (part no: 122-5222UI, Agilent J\&W GC columns). The injector temperature was set to $260{ }^{\circ} \mathrm{C}$, front

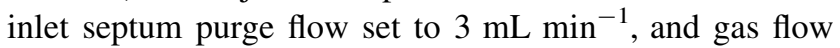
rate through the column set to $1 \mathrm{~mL} \mathrm{~min}^{-1}$. Column temperature was held at $70{ }^{\circ} \mathrm{C}$ for $2 \mathrm{~min}$, then increased by $20{ }^{\circ} \mathrm{C} \mathrm{min}{ }^{-1}$ to $320{ }^{\circ} \mathrm{C}$, and held for $8 \mathrm{~min}$. The column effluent was led into the ion source of a Pegasus HT GCTOF-MS (LECO Corp., St Joseph, MI, USA). The transfer line and ion source temperatures were 270 and $200{ }^{\circ} \mathrm{C}$, respectively. Detector voltage was set to $1650 \mathrm{~V}$. Ions were generated by a $-70 \mathrm{~V}$ electron beam at an ionization current of $2.0 \mathrm{~mA}$, and 20 spectra s$^{-1}$ were recorded in the mass range $50-800 \mathrm{~m} / \mathrm{z}$. The acceleration voltage was turned on after a solvent delay of $270 \mathrm{~s}$.

\subsection{Data processing of samples subjected to GC- TOF-MS}

Unprocessed MS files from GC/TOF-MS analysis were exported in NetCDF format to MATLAB software R2013a (Mathworks, Natick, MA). All data pretreatment procedures, including baseline correction, chromatogram alignment, time-window setting and multivariate curve resolution (MCR) (Jonsson et al. 2005) were performed in MATLAB using custom scripts. Peak detection against mass spectra libraries (targeted data processing) was performed with an in-house script. Metabolites were identified by using NIST MS Search 2.0 software to compare the mass spectra of all detected compounds with spectra in: the NIST library 2.0, the in-house mass spectra library established by Swedish Metabolomics Centre, and the mass spectra library maintained by the Max Planck Institute in Golm (http://csbdb. mpimp-golm.mpg.de/csbdb/gmd/gmd.html).

A retention index comparison was performed, with a retention index deviation $< \pm 10$ (in addition to a high spectral match) resulting in a positive ID. Generated peaks were analysed in tandem using the spectral database found at www.massbank.jp. The data was normalized using all 11 internal standards (eluting over the whole chromatographic time range). A principal component analysis (PCA), using peak areas for the internal standards, was conducted and the T-score value for each sample was used to normalize the resolved data by dividing the peak areas of each sample with its corresponding score value. Multivariate analysis was performed with SIMCA-P +13 software (Umetrics $\mathrm{AB}$, Umeå, Sweden). Data from the analysis and peakspectra is available as (Online Resource 12-13).

\subsection{Data analysis}

Data was preprocessed for an integrity check and transformed into the binary logarithm [base of $2 ; \log (2)]$ for downstream analysis (Stacklies et al. 2007). Extreme outliers were replaced by the median of the data within biological replicates, and data was subjected to pareto scaling (Dieterle et al. 2006). Heatmaps were generated using the heatmap.2 function in the gplots package in $\mathrm{R}$ software with data transformed into the common logarithm [base of $10 ; \log (10)]$ using averages of metabolite levels.

PCA is an unsupervised method for finding the directions that best explain the variance in a data set $(\mathrm{X})$ without referring to classification labels $(\mathrm{Y})$. PCA was performed using the prcomp syntax in R (William N. Venables 2002) or SIMCA, version 14.0.0.1359 (Umetrics AB, Umeå, Sweden).

Partial least-squares discriminant analysis (PLS-DA) is a supervised method that uses multivariate regression techniques to extract information that can predict classification $(\mathrm{Y})$ via linear combination of original variables $(\mathrm{X})$. PLS-DAs were performed on $\log (2)$-transformed metabolite concentrations that had been centered according to the means using SIMCA, version 14.0.0.1359 (Umetrics AB, Umeå, Sweden); unit-variance scaling was applied as previously described (Slupsky et al. 2007).

A permutation test was performed (20 permutations) to assess the significance of classification, and prediction accuracy was determined (Max Kuhn. Contributions from Jed Wing and Steve Weston and Andre Williams. caret: Classification And REgression Training, 2008, R package version 3.45) (Bijlsma et al. 2006). Variable Importance in Projection (VIP) in PLS-DA is a weighted sum of the squares of the PLS loadings that accounts for the amount of explained Y-variation in each dimension for each component (Max Kuhn. Contributions from Jed Wing and Steve Weston and Andre Williams. caret: Classification And Regression Training, 2008).

\section{Results}

\subsection{Loss of $\mathrm{ncm}^{5} \mathrm{U}, \mathrm{mcm}^{5} \mathrm{U}$ and $\mathrm{mcm}^{5} \mathrm{~s}^{2} \mathrm{U}$ wobble uridine nucleosides in tRNA result in an altered metabolic profile}

We subjected an elp $3 \Delta$ strain carrying either an empty low copy LEU2 vector (elp34-1.c.-empty) or the same vector containing the wild-type ELP3 gene (elp3A-1.c.-ELP3) to metabolic profiling using non-targeted GC-TOF-MS (Fig. 1). This metabolic profiling was conducted to investigate whether loss of the $n \mathrm{~cm}^{5} \mathrm{U}, \mathrm{mcm}^{5} \mathrm{U}$ and $\mathrm{mcm}^{5} \mathrm{~s}^{2} \mathrm{U}$ wobble uridine nucleosides in yeast tRNA causes 
Fig. 1 Overview of the pipeline for metabolic profiling of wildtype and elp $3 \Delta$ strains carrying indicated plasmids. The UMY4238 and UMY4239 yeast strains contained either: an empty low-copy pRS315 vector (elp34-l.c.-empty); a pRS315 vector containing the wild-type ELP3 gene (elp34-1.c.-ELP3); an empty high copy pRS425 vector (elp34-h.c.-empty, WTh.c.-empty); or a high copy pRS425 vector carrying the tRNA genes $t K(U U U)$, $t Q(U U G)$ and $t E(U U C)$ (elp3Ah.c.-tKQE, WT-h.c.-tKQE).

Yeast strains were cultivated logarithmically to an $\mathrm{OD}_{600}$ value of $\sim 0.5$ at 30 or $34^{\circ} \mathrm{C}$ and harvested. Metabolites were extracted and then quantified using GC-TOF-MS. Metabolite data was analyzed using multivariate analysis (PCA, PLS-DA) which separated the metabolites according to different classes representing the elp 34 strains containing various plasmid constructs

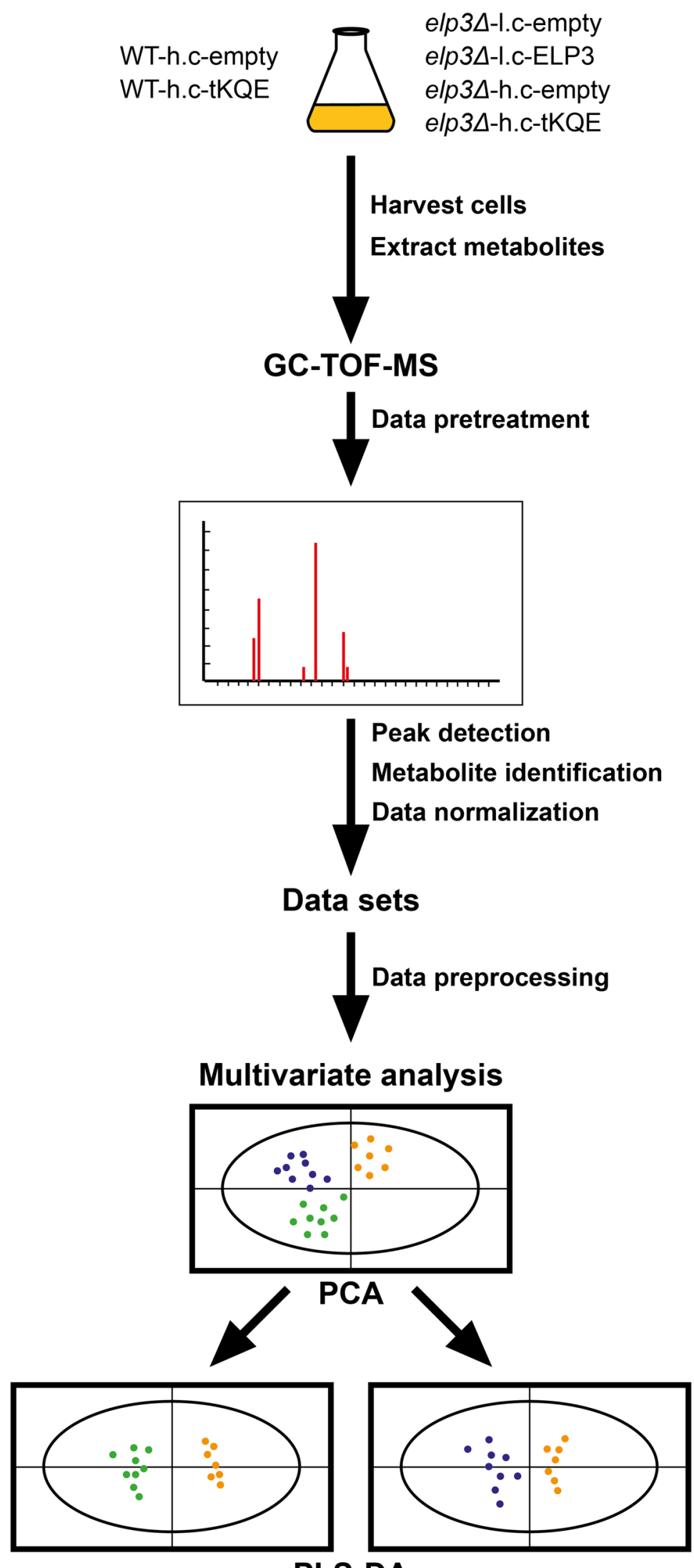

PLS-DA 
metabolic alterations. We also included samples of the wild-type strain carrying an empty high-copy $L E U 2$ vector (WT-h.c.-empty) to investigate whether metabolism of the elp3A-1.c.-ELP3 strain represents that of the wild-type strain.

Since elp $3 \Delta$ strains are temperature sensitive (Ts), we cultured strains to $\sim 0.5 \mathrm{OD}_{600}$ units under permissive $\left(30{ }^{\circ} \mathrm{C}\right)$ and semi-permissive $\left(34{ }^{\circ} \mathrm{C}\right)$ growth conditions, as metabolic changes may be more pronounced at elevated temperatures. From our metabolite extracts, 111 metabolites could be measured using GC-TOF-MS, 41 of which could be identified while the remaining were unidentified. We performed a PCA of all the strains used and all 111 metabolites detected in this study to get an overview of metabolism in the strains. The PCA results showed that the elp3A-1.c.-ELP3 strain clusters with the WT-h.c.-empty strain, indicating that metabolism in these strains is similar (Fig. 2).

We looked for alterations in levels of specific metabolites in the elp3 $\Delta$ strain using partial least squares regression discriminant analysis (PLS-DA) (Figs. 3a-f, 4a-d). Metabolites with a variable importance for the projection (VIP) below one were excluded (Chong and Jun 2005).

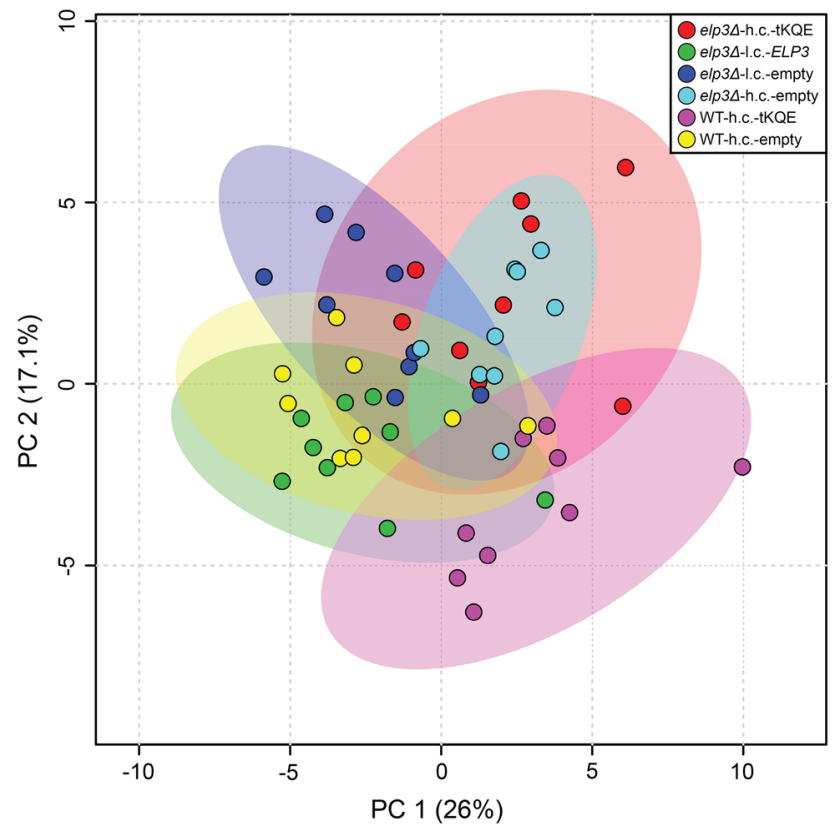

Fig. 2 Metabolic variation of the wild-type and elp34 strain containing indicated plasmids. The UMY4238 and UMY4239 yeast strains containing either: an empty low-copy pRS315 vector (elp3 $\Delta$ 1.c.-empty); a pRS315 vector containing the wild-type ELP3 gene (elp34-1.c.-ELP3); an empty high copy pRS425 vector (elp34-h.c.empty, WT-h.c.-empty); or a high copy pRS425 vector carrying the tRNA genes $t K(U U U), t Q(U U G)$ and $t E(U U C)$ (elp3 4 -h.c.-tKQE, WT-h.c.-tKQE) were grown logarithmically to an $\mathrm{OD}_{600}$ of $\sim 0.5$ at $30{ }^{\circ} \mathrm{C}$ and harvested. Metabolites were extracted and then quantified using GC-TOF-MS. Each dot in the PCA analysis represents a technical replicate from three different biological replicates
Briefly, PLS-DA allows analysis of large sample sets structured in the form of classes. The classes are separated according to a comparison between all variables within one class and all variables within another class, and subsequent prediction of the variables that account for the class separation. Variables that are good predictors for separating one class from another have a high VIP score, while variables with a low VIP score do not contribute to class separation.

A comparison of the metabolic profiles of only identified metabolites showed that permissive growth $\left(30^{\circ} \mathrm{C}\right)$ of the elp34-1.c.-empty strain resulted in elevated levels of primarily Ornithine, Lysine and $N$-Acetylglucosamine, and reduced levels of Glutamine, Beta-alanine, Malic acid, Aspartic acid, Pyroglutamic acid, Alanine, Threonine, 2-Aminobutyric acid, 5,6-Dihydrouracil and Tyrosine when compared to the elp3A-1.c.-ELP3 strain (Fig. 5a-e, Online Resource 2-3). Semi-permissive growth $\left(34{ }^{\circ} \mathrm{C}\right)$ of the elp34-1.c.-empty strain also resulted in elevated levels of Ornithine, Lysine and N-Acetylglucosamine when compared to the elp34-1.c.-ELP3 strain (Fig. 5a-e, Online Resource 2-3). We also observed that the elp3 4 -1.c.-empty strain accumulated eight additional metabolites with known identity at $34{ }^{\circ} \mathrm{C}$ (Online Resource 4), indicating more pronounced metabolic changes with growth at elevated temperatures.

Semi-permissive $\left(34^{\circ} \mathrm{C}\right)$ growth of the elp34-1.c.-empty strain showed reduced levels of Beta-alanine and Glutamine, as observed at permissive growth $\left(30^{\circ} \mathrm{C}\right)(\mathrm{Fig}$. $5 \mathrm{a}-$ e, Online Resource 2-4). In addition, semi-permissive growth of the elp34-1.c.-empty strain showed reduced levels of Malic acid, Aspartic acid and Threonine. Surprisingly, we did not observe significantly reduced levels of Pyroglutamic acid, Alanine, 2-Aminobutyric acid, 5,6-Dihydrouracil and Tyrosine after semi-permissive growth, as when the elp34-1.c.-empty strain grew at $30{ }^{\circ} \mathrm{C}$. Instead, semi-permissive growth of this strain resulted in reduced levels of Gluconic acid 1,4-lactone, Glucose, Trehalose, Octadecanoic acid and Glucuronic acid-E-lactone.

Moreover, we observed that several unidentified metabolites had a VIP score above one, indicating altered metabolism of these metabolites in the elp3A-1.c.-empty strain compared to the elp34-1.c.-ELP3 strain at both 30 and $34{ }^{\circ} \mathrm{C}$ (Online Resource 5-6). The amounts of identified and unidentified metabolites with altered levels in the elp3A-1.c.-empty strain and the elp3A-1.c.-ELP3 strain were higher when these strains grew at $34{ }^{\circ} \mathrm{C}$ (Online Resource 5-6). Our results show that semi-permissive growth of the elp34-1.c.-empty strain resulted in more metabolic alterations than permissive growth. More metabolites started to increase or decrease at $34{ }^{\circ} \mathrm{C}$ in the elp34-1.c.-empty strain while levels of these metabolites were more or less constant at both 30 and $34{ }^{\circ} \mathrm{C}$ in the elp3A-1.c.-ELP3 strain. 
A
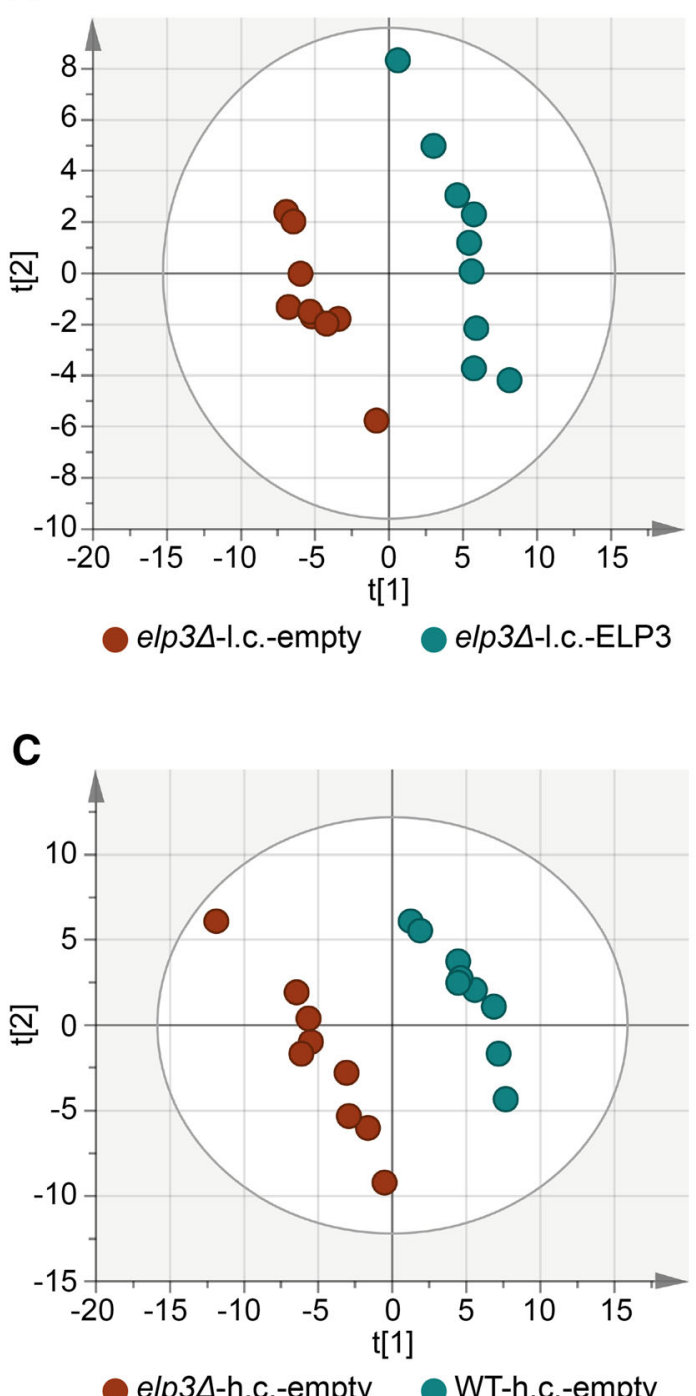

E

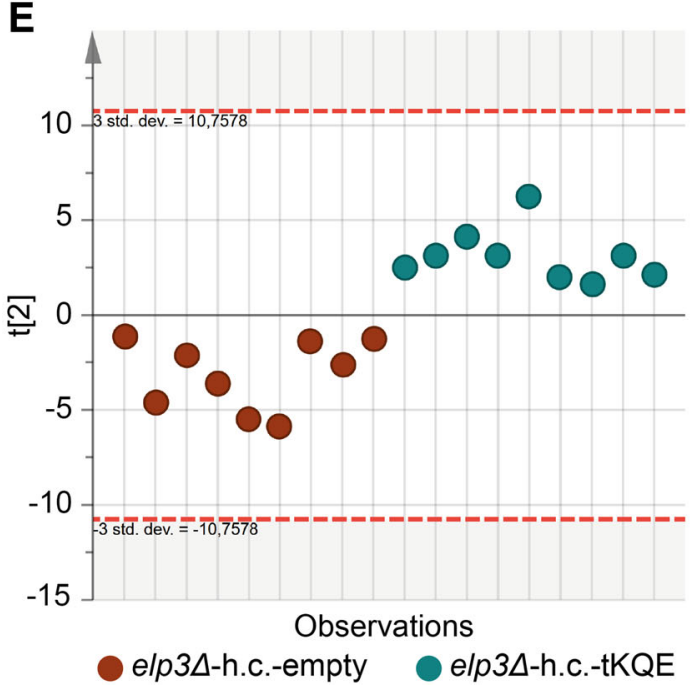

B

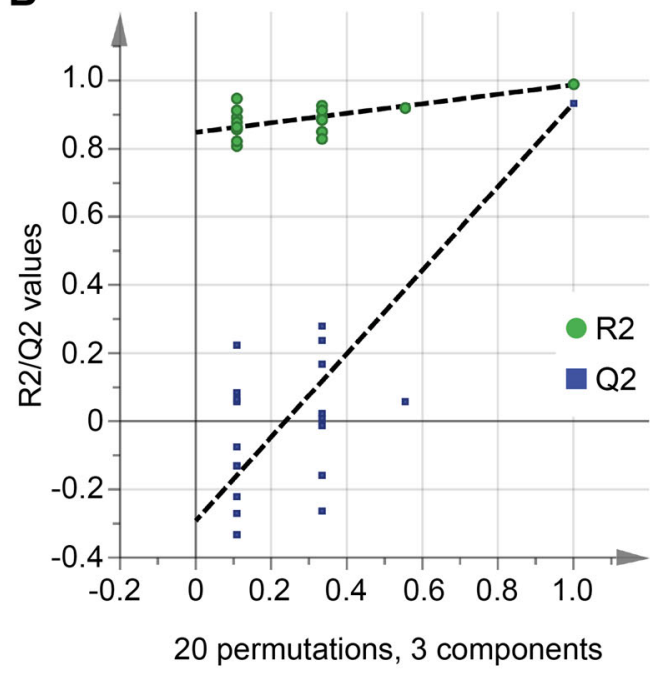

D

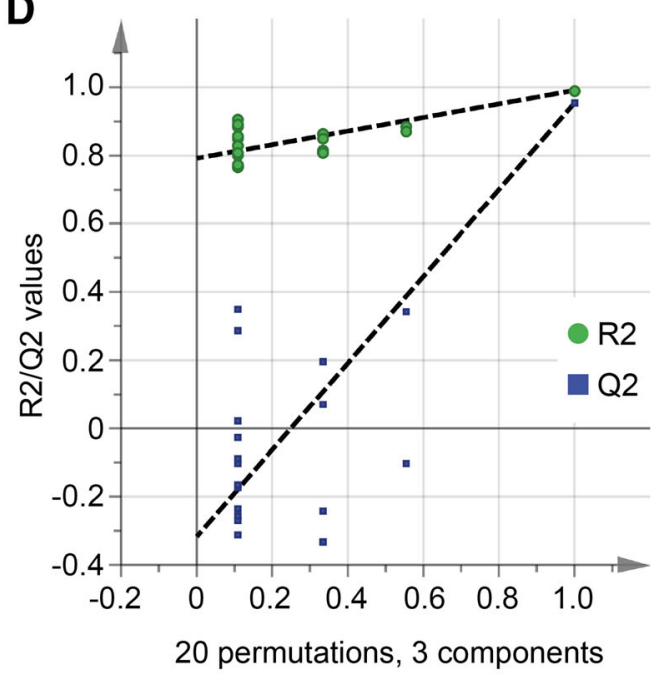

F

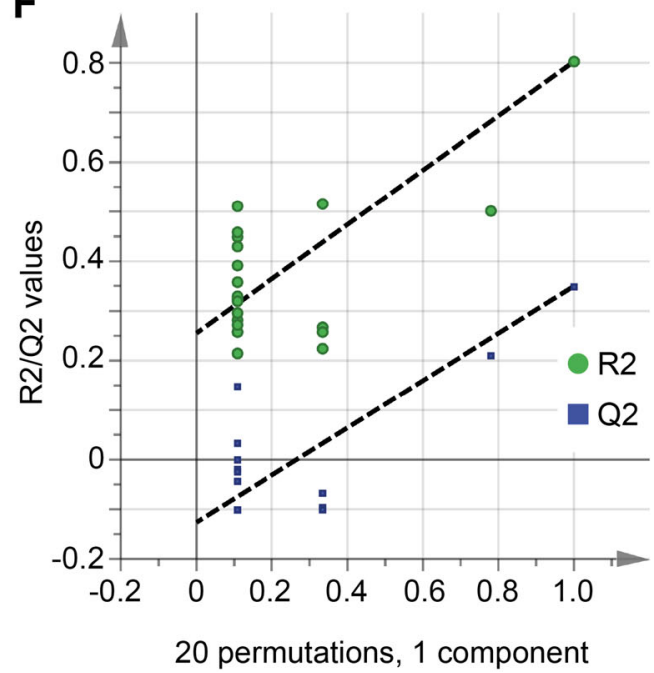


4Fig. 3 Score plots summarizing the PLS-DA modelling of strains grown at $30{ }^{\circ} \mathrm{C}$. a PLS-DA score plot when modelling the elp3 $\Delta$ strain with an empty low copy pRS315 vector (elp3A-1.c.-empty) against the elp3A strain containing the wild-type ELP3 gene on a pRS315 vector (elp34-1.c.-ELP3). b Random permutation (20 randomizations) testvalidation plot of PLS-DA model in (a). c PLS-DA score plot when modelling the elp3 $\Delta$ strain with an empty high copy pRS425 vector (elp34-h.c.-empty) against the wild-type strain with an empty pRS425 vector (WT-h.c.-empty). d Random permutation (20 randomizations) test-validation plot of PLS-DA model in (c). e PLS-DA score plot when modelling the elp3 $\Delta$ strain with an empty high copy pRS425 vector (elp34-h.c.-empty) against the elp3 $\Delta$ strain containing a pRS425 vector carrying the tRNA genes $t K(U U U), t Q(U U G)$ and $t E(U U C)$ (elp3 $\Delta$-h.c.-tKQE). f Random permutation (20 randomizations) test-validation plot of PLS-DA model in (e). The PLS-DA model in e only has one valid component and is therefore portrayed by one vector. Each dot in $\mathbf{a}, \mathbf{c}$ and $\mathbf{e}$ represents a technical replicate from three different biological replicates

\subsection{Elevated levels of hypomodified tRNA ${ }_{s^{2} U U U}^{\mathrm{Lys}}$, tRNA $_{\mathrm{s}^{2} \mathrm{UUG}}^{\mathrm{Gln}}$ and tRNA $\mathrm{s}^{2} \mathrm{UUC}$ partially suppress certain metabolic alterations}

All phenotypes tested in yeast Elongator mutants to date have been partially suppressed by elevated levels of various combinations of hypomodified tRNA $\mathrm{s}_{\mathrm{s}^{2} \mathrm{UUU}}^{\mathrm{Lys}}, \mathrm{tRNA}_{\mathrm{s}^{2} \mathrm{UUG}}^{\mathrm{Gln}}$ and

Fig. 4 Score plots summarizing the PLS-DA modelling of strains grown at $34{ }^{\circ} \mathrm{C}$. a PLSDA score plot when modelling the elp3 $\Delta$ strain with an empty low copy pRS315 vector (elp3A-1.c.-empty) against the elp3 $\triangle$ strain containing the wild-type $E L P 3$ gene on a pRS315 vector (elp34-1.c.ELP3). b Random permutation (20 randomizations) testvalidation plot of PLS-DA model in a. c PLS-DA score plot when modelling the elp3 4 strain with an empty high copy pRS425 vector (elp34-h.c.empty) against the elp3 $\Delta$ strain containing a pRS425 vector carrying the tRNA genes $t K(U U U), t Q(U U G)$ and $t E(U U C)$ (elp3 $\Delta$-h.c.-tKQE). d Random permutation (20 randomizations) test-validation plot of PLS-DA model in (c). Each dot in a, c and e represents a technical replicate from three different biological replicates
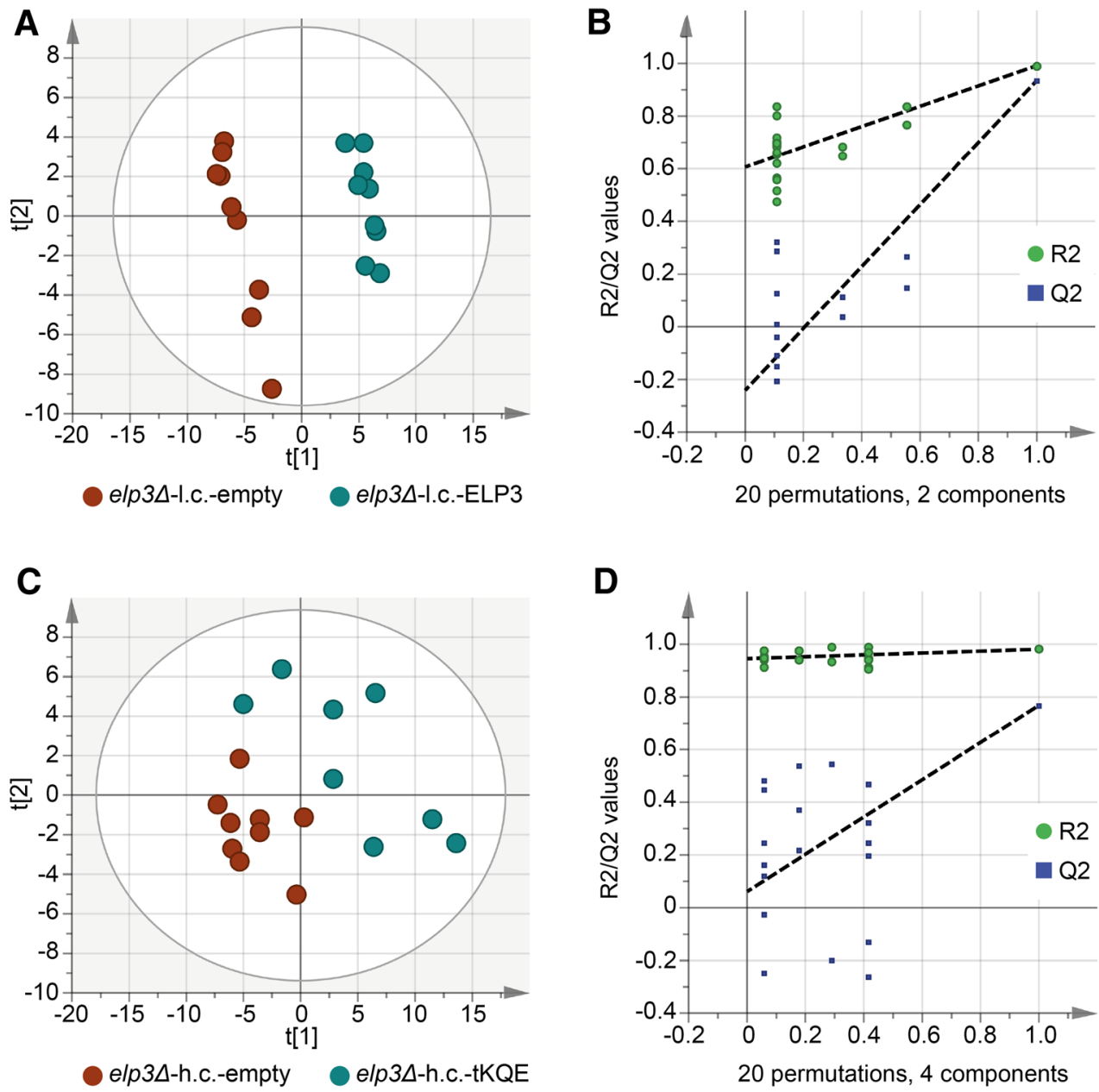

tRNA $\mathrm{s}^{2} \mathrm{UUU}$ (Esberg et al. 2006; Chen et al. 2011; Tigano et al. 2015; Fernandez-Vazquez et al. 2013; Bauer et al. 2012; Nedialkova and Leidel 2015). Thus, we were interested in investigating whether the metabolic alterations in elp3 4 cells are suppressed by elevated levels of tRNA $_{\mathrm{s}^{2} \mathrm{UUU}}^{\mathrm{Lys}}, \mathrm{tRNA}_{\mathrm{s}^{2} \mathrm{UUG}}^{\mathrm{Gln}}$ and tRNA $\mathrm{s}^{2} \mathrm{UUC}$. We compared the PLS-DA model of the elp3 $\Delta$ strain that either contained an empty pRS425 high-copy vector (elp3A-h.c.-empty) or overexpressed tRNA ${ }_{\mathrm{s}^{2} \mathrm{UUU}}^{\text {Lys }}, \mathrm{tRNA}_{\mathrm{s}^{2} \mathrm{UUG}}^{\mathrm{Gln}}$ and tRNA $\mathrm{s}^{2} \mathrm{UUC}$ (elp3A-h.c.-tKQE) with the PLS-DA model that compared the elp3A-1.c.-empty strain with the elp3A-1.c.-ELP3 strain.

PLS-DA modelling of the comparison between the elp3 $\Delta$-h.c.-empty strain and the elp3 $\Delta$-h.c.-tKQE strain produced models with lower $\mathrm{Q}^{2}$-values than PLS-DA models of the comparison between the elp3 $\triangle$-l.c.-empty and elp34-1.c.-ELP3 strains (Figs. 3a-f, 4a-d, Online Resource 7). This result indicates that very few metabolic alterations are suppressed by overexpression of

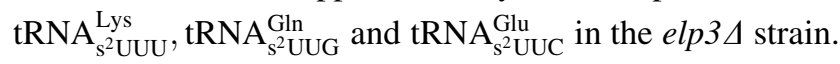
However, the growth defect of the elp3 $\Delta$ strain at $34{ }^{\circ} \mathrm{C}$ was partially suppressed by overexpression of the aforementioned tRNAs (data not shown). 
A

Color key
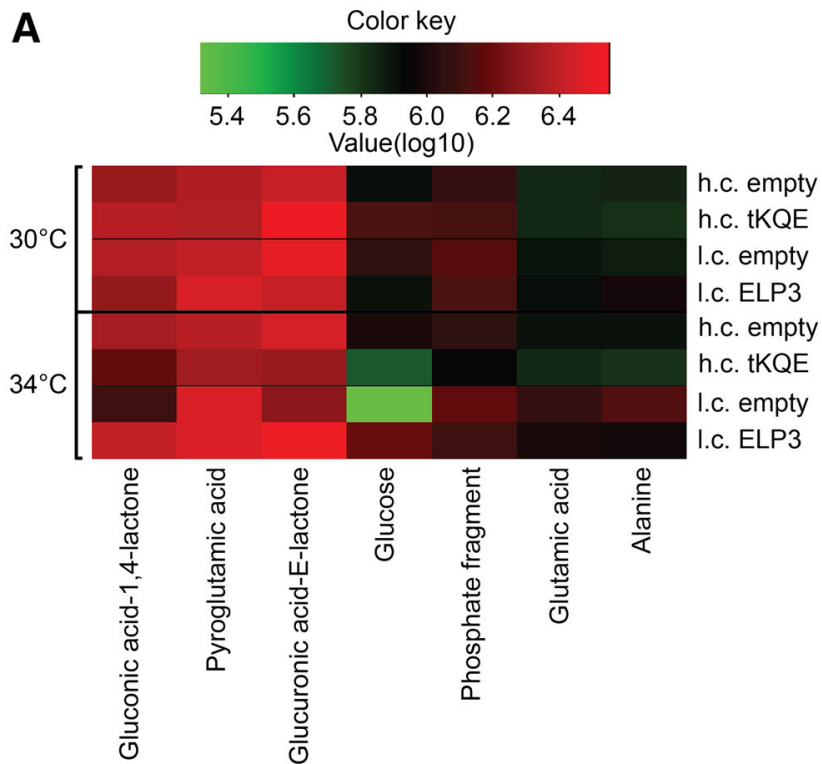

C
$30^{\circ} \mathrm{C}$
$34^{\circ} \mathrm{C}$

Color key
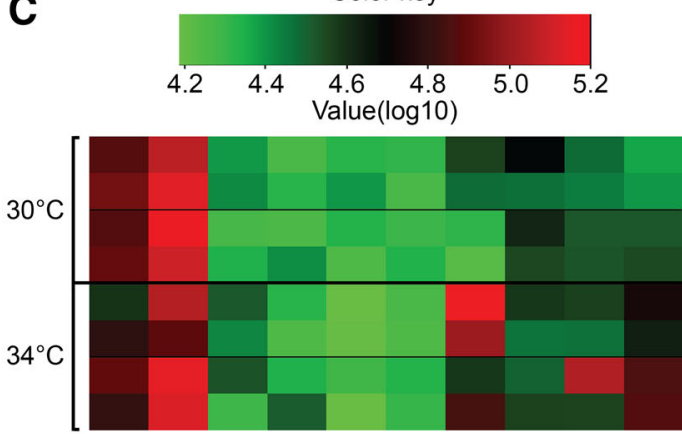

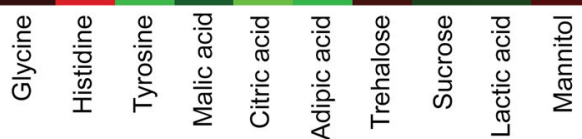

E

Color key

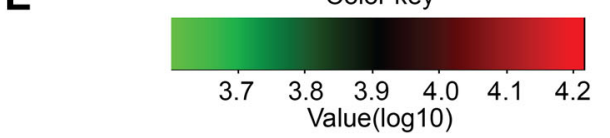

Value $(\log 10)$

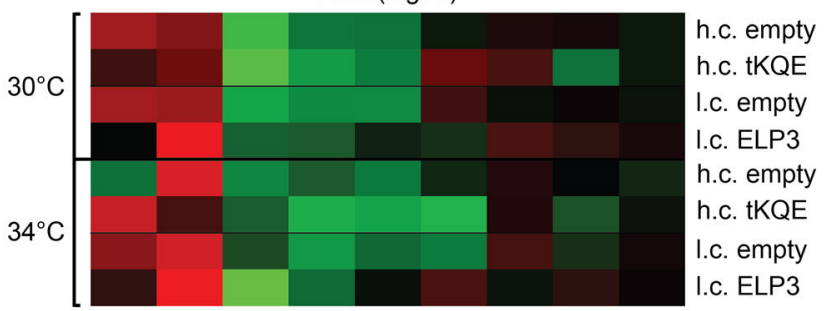

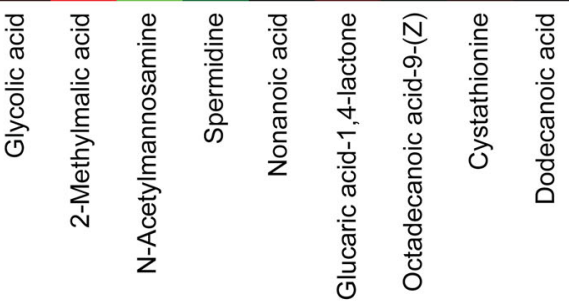
I.c. empty

h.c. empty h.c. $\mathrm{tKQE}$ I.c. empty I.c. ELP3 h.c. empty h.c. $\mathrm{tKQE}$ I.c. empty I.c. ELP3

h.c. empty h.c. $\mathrm{K} Q \mathrm{E}$ l.c. empty l.c. ELP3

h.c. empty l.c. empty I.c. ELP3

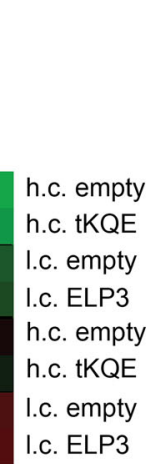

h.c. empty h.c. $\mathrm{K} Q \mathrm{E}$ I.c. empty I.c. ELP3 h.c. empty h.c. $t K Q E$ I.c. empty I.c. ELP3 .

B

h.c. empty h.c. $\mathrm{K} K \mathrm{EE}$ I.c. ELP3 h.c. empty h.c. $\mathrm{K} K Q \mathrm{E}$ I.c. empty I.c. ELP3 $34^{\circ} \mathrm{C}$
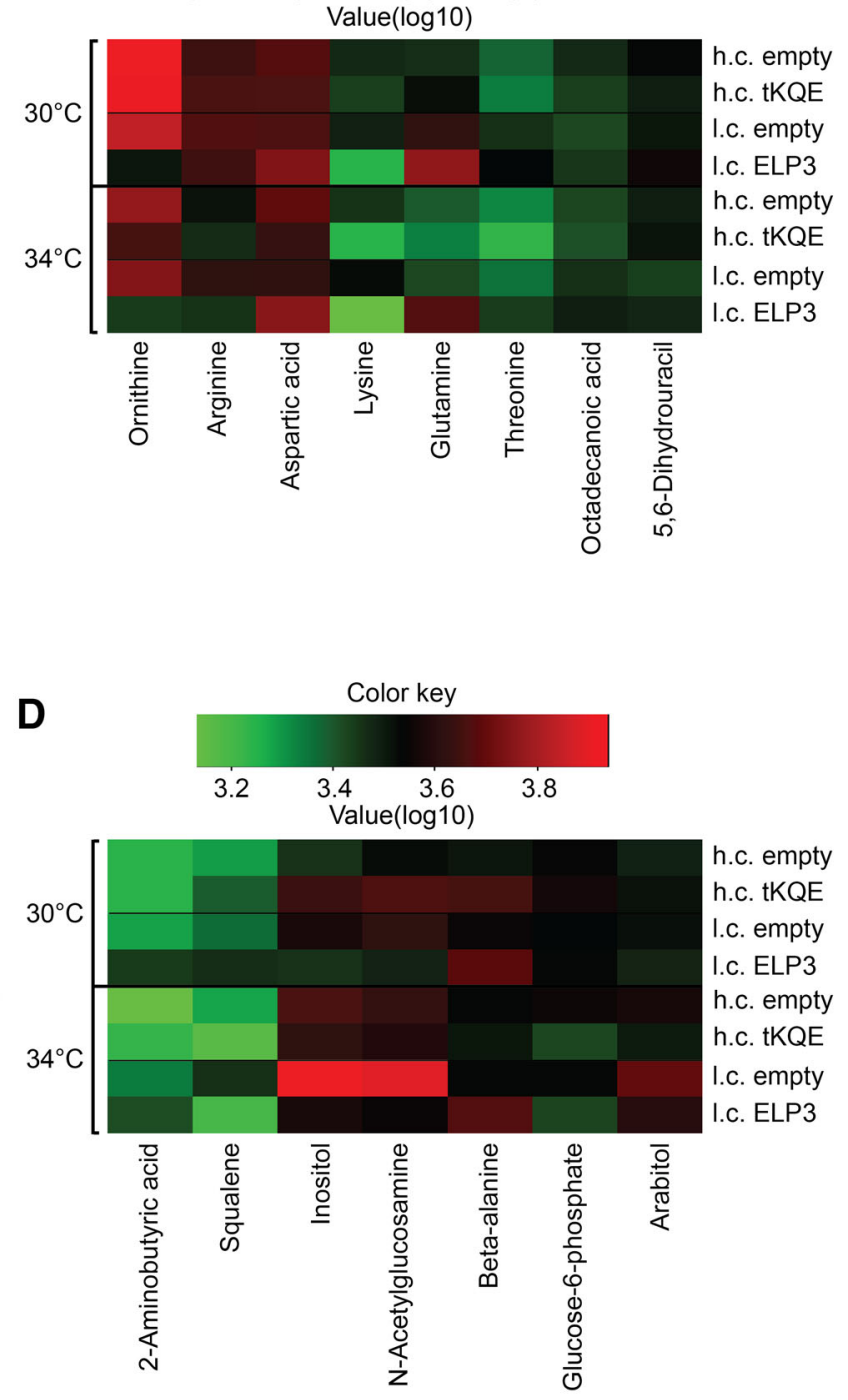

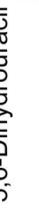


4Fig. 5 Metabolic alterations upon loss of $\mathrm{ncm}^{5} \mathrm{U}, \mathrm{mcm}^{5} \mathrm{U}$ and $\mathrm{mcm}^{5} \mathrm{~s}^{2} \mathrm{U}$ wobble uridine nucleosides in tRNA. The UMY4239 elp3 4 strains contained either: an empty pRS315 low copy vector (1.c. empty); a pRS315 vector carrying the wild-type ELP3 gene (1.c. ELP3); an empty pRS425 high copy vector (h.c. empty); or a pRS425 high copy vector carrying the tRNA genes $t K(U U U) ; t Q(U U G)$ and $t E(U U C)$ (h.c. $\mathrm{tKQE})$. These yeast strains were grown logarithmically to $\sim 0.5 \mathrm{OD}_{600}$ at 30 or $34{ }^{\circ} \mathrm{C}$ and harvested. Metabolites were extracted and then quantified using GC-TOF-MS. Metabolites were hierarchically organized into five clusters as represented by $\mathbf{a}-\mathbf{e}$ based on metabolite abundance (see Online Resource 2). Red signifies metabolite enrichment and green signifies metabolite reduction

At permissive $\left(30^{\circ} \mathrm{C}\right)$ growth we observed suppression of alteration of beta-alanine metabolism, and weak suppression of alterations of Glutamine, Tyrosine, Ornithine and Lysine metabolism from a comparison between metabolic patterns of identified metabolites from the elp3A-h.c.-empty and elp3 4 -h.c.-tKQE strains grown at $30{ }^{\circ} \mathrm{C}$. We did not observe suppression of the altered metabolism of: Malic acid, Aspartic acid, Pyroglutamic acid, Alanine, Threonine, 2-Aminobutyric acid, 5,6-Dihydrouracil and $\mathrm{N}$-Acetylglucosamine (Fig. 5a-e, Online Resource 2, 8).

Next, we investigated whether overexpression of tRNA $_{\mathrm{s}^{2} \mathrm{UUU}}^{\mathrm{Lys}}, \mathrm{tRNA}_{\mathrm{s}^{2} \mathrm{UUG}}^{\mathrm{Gl}}$ and tRNA $\mathrm{s}^{2} \mathrm{UUC}$ in the elp $3 \Delta$ strain led to a unique suppression pattern of metabolic alterations during growth at $34{ }^{\circ} \mathrm{C}$. A comparison between the metabolite patterns of the elp3 3 -h.c.-empty and elp3 4 -h.c.tKQE strains grown at $34{ }^{\circ} \mathrm{C}$ revealed that elevated levels of $\mathrm{tRNA}_{\mathrm{s}^{2} \mathrm{UUU}}^{\mathrm{Lys}}, \mathrm{tRNA}_{\mathrm{s}^{2} \mathrm{GUG}}^{\mathrm{Gln}}$ and $\mathrm{tRNA} \mathrm{s}^{2} \mathrm{GUC}$ suppressed alterations in Lysine and Ornithine metabolism. However, changes in Beta-alanine and Glutamine metabolism were not suppressed in elp34-h.c.-tKQE strains grown at $34{ }^{\circ} \mathrm{C}$. Elevated levels of tRNA $\mathrm{s}_{\mathrm{s}^{2} \mathrm{UUU}}^{\mathrm{Ly}}, \mathrm{tRNA}_{\mathrm{s}^{2} \mathrm{UUG}}^{\mathrm{Gln}}$ and tRNA $\mathrm{s}^{2} \mathrm{GuC}$ partially suppressed alterations in Lactic acid, Tyrosine, Alanine and Glutamic acid metabolism at $34{ }^{\circ} \mathrm{C}$. Moreover, an analytical trend indicated that alterations in several other metabolites may be weakly suppressed (Fig. 5a-e, Online Resource 2, 9).

Several unidentified metabolites were suppressed in the elp3 4 strains at both 30 and $34{ }^{\circ} \mathrm{C}$ (Online Resource 1011). Overall, our results indicate that suppression of certain metabolic alterations varies with elevated levels of tRNA $_{\mathrm{s}^{2} \mathrm{UUU}}^{\mathrm{Lys}}, \mathrm{tRNA}_{\mathrm{s}^{2} \mathrm{UUG}}^{\mathrm{Gln}}$ and tRNA $\mathrm{s}^{2} \mathrm{GUC}$ in the elp $3 \Delta$ strain depending on the growth temperature. However, Ornithine and Lysine are exceptions in the elp $3 \Delta$ strain as alterations of these metabolites are partially suppressed with elevated levels of the three tRNAs at both 30 and $34{ }^{\circ} \mathrm{C}$.

\section{Discussion}

Mutations in genes encoding Elongator complex subunits have been linked to a multitude of phenotypes in S. cerevisiae (Otero et al. 1999; Wittschieben et al. 1999; Winkler et al. 2002; Rahl et al. 2005; Tigano et al. 2015; Nedialkova and Leidel 2015; Frohloff et al. 2001; Chen et al. 2011; Q. Li et al. 2009). Many investigations in Eukaryotes support a role for the complex in formation of the $\mathrm{ncm}^{5} \mathrm{U}$, $\mathrm{mcm}{ }^{5} \mathrm{U}$ and $\mathrm{mcm}^{5} \mathrm{~s}^{2} \mathrm{U}$ wobble uridine nucleosides in tRNA [Reviewed in (Karlsborn et al. 2014b)]. Furthermore, phenotypes observed in yeast Elongator mutants can be suppressed by overexpression of various combinations of $\mathrm{tRNA}_{\mathrm{s}^{2} \mathrm{UUU}}^{\mathrm{Lys}}, \mathrm{tRNA}_{\mathrm{s}^{2} \mathrm{UUG}}^{\mathrm{Gln}}$ and tRNA $\mathrm{s}_{\mathrm{s}^{2} \mathrm{UUC}}^{\mathrm{Glu}}$ (Esberg et al. 2006; Chen et al. 2011; Tigano et al. 2015; FernandezVazquez et al. 2013; Bauer et al. 2012; Nedialkova and Leidel 2015). This suppression has been ascribed to restoring translational efficiency of codons normally read by $\mathrm{tRNA}_{\mathrm{mcm}^{5} \mathrm{~s}^{2} \mathrm{UUU}}^{\mathrm{Lys}}$, tRNA $\mathrm{mcm}_{\mathrm{m} \mathrm{s}^{2} \mathrm{UUG}}^{\mathrm{Gln}}$ and tRNA $\mathrm{mcm}_{\mathrm{m} \mathrm{s}^{2} \mathrm{UUC}}^{\mathrm{Glu}}$ by compensating the reduced codon-anticodon interaction of the hypomodified tRNAs with elevated levels of these three tRNA species (Esberg et al. 2006; Chen et al. 2011).

In this study, we used untargeted GC-TOF-MS based metabolomics to investigate the extent of metabolic alterations in an elp3 3 strain. We found that loss of the $\mathrm{ncm}^{5} \mathrm{U}$, $\mathrm{mcm}^{5} \mathrm{U}$ and $\mathrm{mcm}^{5} \mathrm{~s}^{2} \mathrm{U}$ wobble uridine nucleosides in tRNA resulted in an altered metabolism, and that only a subset of these alterations were suppressed by overexpression of $\mathrm{tRNA}_{\mathrm{s}^{2} \mathrm{UUU}}^{\mathrm{Lys}}, \mathrm{tRNA}_{\mathrm{s}^{2} \mathrm{UUG}}^{\mathrm{Gln}}$ and tRNA $\mathrm{s}_{\mathrm{s}^{2} U U C}^{\mathrm{Glu}}$. This was surprising, as all phenotypes tested in yeast elp $3 \Delta$ strains to date, except the tRNA modification defect, have been at least partially suppressed by elevated levels of various combinations of these three tRNAs (Esberg et al. 2006; Chen et al. 2011; Tigano et al. 2015; Fernandez-Vazquez et al. 2013; Bauer et al. 2012). Therefore, suppression of most of the metabolic alterations observed in an elp $3 \Delta$ mutant may require elevated levels of additional tRNA species that normally have the $n \mathrm{~cm}^{5} \mathrm{U}, \mathrm{mcm}^{5} \mathrm{U}$ and $\mathrm{mcm}^{5} \mathrm{~s}^{2} \mathrm{U}$ wobble uridine nucleosides.

It is possible that some metabolic alterations in the elp3 $\Delta$ strain could be transient, or observed only when cells are exposed to certain stress conditions. Furthermore, metabolic alterations observed in the elp $3 \Delta$ strain could, in part, be a global metabolic adaptation of the primary metabolic defects due to inefficient translation. If elp3 $\Delta$ cells adapt to specific metabolic defects by reconfiguring global metabolism, the stress tolerance may be influenced, as the altered metabolism may cause the cell to be in an unfavourable state of metabolic homeostasis. Thus, overexpression of $\mathrm{tRNA}_{\mathrm{s}^{2} \mathrm{UUU}}^{\mathrm{Lys}}, \mathrm{tRNA}_{\mathrm{s}^{2} \mathrm{UUG}}^{\mathrm{Gln}}$ and $\mathrm{tRNA}_{\mathrm{s}^{2} \mathrm{UUC}}^{\mathrm{Glu}}$ in the elp $3 \Delta$ strain could suppress certain primary metabolic defects and alter the metabolic homeostasis into a more favourable state, resulting in a cellular metabolism better equipped to handle sudden changes in cell physiology due to stress exposure. To differentiate between primary and secondary metabolic defects in the elp3A strain, an instantaneous elimination of the $\mathrm{ncm}^{5} \mathrm{U}, \mathrm{mcm}^{5} \mathrm{U}$ and 
mcm ${ }^{5} \mathrm{~s}^{2} \mathrm{U}$ tRNA modifications is required. However, there are no known enzymes that specifically remove these modifications. Moreover, the long half-life of tRNAs means that a rapid depletion of Elongator would not generate an immediate loss of the modifications, but instead a gradual depletion of modified tRNA species during cellular growth.

Ribosomal profiling studies of Elongator mutants have shown increased ribosome pausing when lysine-AAA and glutamine-CAA codons are in the ribosomal A-site (Nedialkova and Leidel 2015). Increased ribosome pausing in an elp6 4 mutant can be alleviated by overexpression of tRNA $_{\mathrm{s}^{2} \mathrm{UUU}}^{\mathrm{Lys}}, \mathrm{tRNA}_{\mathrm{s}^{2} \mathrm{UUG}}^{\mathrm{Gln}}$ and tRNA $\mathrm{s}^{2} \mathrm{GUC}$ which lack the $\mathrm{mcm}^{5}$ - side chain (Nedialkova and Leidel 2015). These results indicate that global translation efficiency is affected by loss of these side chains in tRNA, and further, that defective translation due to increased ribosome pausing is suppressed by overexpression of the aforementioned tRNA species. Nonetheless, even if ribosomal pausing occurs on AAA and CAA codons, it is possible that only a few mRNAs have translation defects which result in altered protein expression and likely an altered metabolism.

\section{Concluding remarks}

Overall, our metabolic profiling data shows that elp3 $\Delta$ strains have widespread metabolic alterations. These metabolic alterations can be restored by complementation of the elp3 $\triangle$ strains with the wild type ELP3, whereas only a few metabolic alterations are suppressed by overexpression of tRNA $_{\mathrm{s}^{2} \mathrm{UUU}}^{\mathrm{Lys}}, \mathrm{tRNA}_{\mathrm{s}^{2} \mathrm{UUG}}^{\mathrm{Gln}}$ and tRNA $\mathrm{s}^{2} \mathrm{UUC}$. Our metabolic profiling also revealed unidentified metabolites which are altered in the elp3 $\Delta$ strain. In the future, more comprehensive databases over yeast metabolites could allow identification of these metabolites, making it possible for our data set to be of valuable use in future studies of the Elongator complex in Saccharomyces cerevisiae.

Acknowledgments We thank Drs. Marcus Johansson, Hans Stenlund, Anders Nordström and Thomas Moritz for comments on the manuscript and the Swedish metabolomics center for help in analyzing metabolites by GC-TOF-MS.

Funding Anders S. Byström is supported by grants from the Swedish Cancer Foundation (13 0301), Swedish Research Council (621-20123576), Karin and Harald Silvanders Foundation and Insamlingsstiftelsen Umeå University.

\section{Compliance with ethical standards}

Conflict of interest Author Anders S. Byström declares that he has no conflict of interest. Author Tony Karlsborn declares that he has no conflict of interest. Author A K M Firoj Mahmud declares that he has no conflict of interest. Author Hasan Tükenmez declares that he has no conflict of interest.

Ethical approval This article does not contain any studies with human participants or animals performed by any of the authors.

Open Access This article is distributed under the terms of the Creative Commons Attribution 4.0 International License (http://creative commons.org/licenses/by/4.0/), which permits unrestricted use, distribution, and reproduction in any medium, provided you give appropriate credit to the original author(s) and the source, provide a link to the Creative Commons license, and indicate if changes were made.

\section{References}

Bauer, F., Matsuyama, A., Candiracci, J., Dieu, M., Scheliga, J., Wolf, D. A., et al. (2012). Translational control of cell division by Elongator. [Research Support, Non-U.S. Gov't]. Cell Reports, 1(5), 424-433. doi:10.1016/j.celrep.2012.04.001.

Bijlsma, S., Bobeldijk, I., Verheij, E. R., Ramaker, R., Kochhar, S., Macdonald, I. A., et al. (2006). Large-scale human metabolomics studies: a strategy for data (pre-) processing and validation. Analytical Chemistry, 78(2), 567-574. doi:10.1021/ac051495j.

Burke, D., Dawson, D., \& Stearns, T. (2000). Methods in Yeast Genetics. Cold Spring Harbor, NY: Cold Spring Harbor Laboratory Press.

Chen, C., Huang, B., Eliasson, M., Ryden, P., \& Byström, A. S. (2011). Elongator complex influences telomeric gene silencing and DNA damage response by its role in wobble uridine tRNA modification. [Research Support, Non-U.S. Gov't]. PLoS Genetics, 7(9), e1002258. doi:10.1371/journal.pgen.1002258.

Chen, C., Tuck, S., \& Byström, A. S. (2009). Defects in tRNA modification associated with neurological and developmental dysfunctions in Caenorhabditis elegans elongator mutants. PLoS Genetics, 5(7), e1000561. doi:10.1371/journal.pgen.1000561.

Chong, I.-G., \& Jun, C.-H. (2005). Performance of some variable selection methods when multicollinearity is present. Chemometrics and Intelligent Laboratory Systems, 78(1-2), 103-112.

Christianson, T. W., Sikorski, R. S., Dante, M., Shero, J. H., \& Hieter, P. (1992). Multifunctional yeast high-copy-number shuttle vectors. Gene, 110(1), 119-122.

Dieterle, F., Ross, A., Schlotterbeck, G., \& Senn, H. (2006). Probabilistic quotient normalization as robust method to account for dilution of complex biological mixtures. Application in $1 \mathrm{H}$ NMR metabonomics. Analytical Chemistry, 78(13), 4281-4290. doi:10.1021/ac051632c.

Durant, P. C., Bajji, A. C., Sundaram, M., Kumar, R. K., \& Davis, D. R. (2005). Structural effects of hypermodified nucleosides in the Escherichia coli and human tRNA(Lys) anticodon loop: The effect of nucleosides $\mathrm{s}(2) \mathrm{U}, \mathrm{mcm}(5) \mathrm{U}, \mathrm{mcm}(5) \mathrm{s}(2) \mathrm{U}, \mathrm{mnm}(5) \mathrm{s}(2) \mathrm{U}$, $\mathrm{t}(6) \mathrm{A}$, and $\mathrm{ms}(2) \mathrm{t}(6)$ A. Biochemistry, 44(22), 8078-8089.

Esberg, A., Huang, B., Johansson, M. J., \& Byström, A. S. (2006). Elevated levels of two tRNA species bypass the requirement for elongator complex in transcription and exocytosis. Molecular Cell, 24(1), 139-148.

Fernandez-Vazquez, J., Vargas-Perez, I., Sanso, M., Buhne, K., Carmona, M., Paulo, E., et al. (2013). Modification of tRNA(Lys) UUU by elongator is essential for efficient translation of stress mRNAs. PLoS Genetics, 9(7), e1003647. doi:10. 1371/journal.pgen.1003647.

Frohloff, F., Fichtner, L., Jablonowski, D., Breunig, K. D., \& Schaffrath, R. (2001). Saccharomyces cerevisiae Elongator 
mutations confer resistance to the Kluyveromyces lactis zymocin. EMBO Journal, 20(8), 1993-2003.

Gietz, R. D., \& Schiestl, R. H. (2007). High-efficiency yeast transformation using the LiAc/SS carrier DNA/PEG method. Nature Protocols, 2(1), 31-34. doi:10.1038/nprot.2007.13.

Hawkes, N. A., Otero, G., Winkler, G. S., Marshall, N., Dahmus, M. E., Krappmann, D., et al. (2002). Purification and characterization of the human elongator complex. Journal of Biological Chemistry, 277(4), 3047-3052.

Huang, B., Johansson, M. J. O., \& Byström, A. S. (2005). An early step in wobble uridine tRNA modification requires the Elongator complex. RNA, 11(4), 424-436.

Johansson, M. J. O., Esberg, A., Huang, B., Björk, G. R., \& Byström, A. S. (2008). Eukaryotic wobble uridine modifications promote a functionally redundant decoding system. Molecular and Cellular Biology, 28(10), 3301-3312.

Jonsson, P., Johansson, A. I., Gullberg, J., Trygg, J., A, J., Grung, B., et al. (2005). High-throughput data analysis for detecting and identifying differences between samples in GC/MS-based metabolomic analyses. Analytical Chemistry, 77(17), 5635-5642. doi:10.1021/ac050601e.

Karlsborn, T., Tükenmez, H., Chen, C., \& Byström, A. S. (2014a). Familial dysautonomia (FD) patients have reduced levels of the modified wobble nucleoside mcmsU in tRNA. Biochemical and Biophysical Research Communications, 454(3), 441-445. doi:10.1016/j.bbrc.2014.10.116.

Karlsborn, T., Tükenmez, H., Mahmud, A. K., Xu, F., Xu, H., \& Byström, A. S. (2014b). Elongator, a conserved complex required for wobble uridine modifications in eukaryotes. RNA Biology, 11(12), 1519-1528. doi:10.4161/15476286.2014. 992276.

Krogan, N. J., \& Greenblatt, J. F. (2001). Characterization of a sixsubunit holo-elongator complex required for the regulated expression of a group of genes in Saccharomyces cerevisiae. Molecular and Cellular Biology, 21(23), 8203-8212.

Li, Q., Fazly, A. M., Zhou, H., Huang, S., Zhang, Z., \& Stillman, B. (2009). The elongator complex interacts with PCNA and modulates transcriptional silencing and sensitivity to DNA damage agents. PLoS Genetics, 5(10), e1000684. doi:10.1371/ journal.pgen.1000684.

Li, Y., Takagi, Y., Jiang, Y., Tokunaga, M., Erdjument-Bromage, H., Tempst, P., et al. (2001). A multiprotein complex that interacts with RNA polymerase II elongator. Journal of Biological Chemistry, 276(32), 29628-29631.

Lin, F. J., Shen, L., Jang, C. W., Falnes, P. O., \& Zhang, Y. (2013). Ikbkap/Elp1 deficiency causes male infertility by disrupting meiotic progression. PLoS Genetics, 9(5), e1003516. doi:10. 1371/journal.pgen.1003516.

Lu, J., Huang, B., Esberg, A., Johansson, M. J. O., \& Byström, A. S. (2005). The Kluyveromyces lactis $\gamma$-toxin targets tRNA anticodons. RNA, 11(11), 1648-1654.

Mehlgarten, C., Jablonowski, D., Wrackmeyer, U., Tschitschmann, S., Sondermann, D., Jäger, G., et al. (2010). Elongator function in tRNA wobble uridine modification is conserved between yeast and plants. Molecular Microbiology, doi:10.1111/j.1365-2958. 2010.07163.x.

Nedialkova, D. D., \& Leidel, S. A. (2015). Optimization of Codon Translation Rates via tRNA Modifications Maintains Proteome Integrity. Cell, 161(7), 1606-1618. doi:10.1016/j.cell.2015.05. 022 .

Nelissen, H., Fleury, D., Bruno, L., Robles, P., De Veylder, L., Traas, J., et al. (2005). The elongata mutants identify a functional Elongator complex in plants with a role in cell proliferation during organ growth. Proceedings of the National Academy of Sciences of the United States of America, 102(21), 7754-7759.
Otero, G., Fellows, J., Li, Y., de Bizemont, T., Dirac, A. M., Gustafsson, C. M., et al. (1999). Elongator, a multisubunit component of a novel RNA polymerase II holoenzyme for transcriptional elongation. Molecular Cell, 3(1), 109-118.

Rahl, P. B., Chen, C. Z., \& Collins, R. N. (2005). Elp1p, the yeast homolog of the FD disease syndrome protein, negatively regulates exocytosis independently of transcriptional elongation. Molecular Cell, 17(6), 841-853.

Rezgui, V. A., Tyagi, K., Ranjan, N., Konevega, A. L., Mittelstaet, J., Rodnina, M. V., et al. (2013). tRNA tKUUU, tQUUG, and tEUUC wobble position modifications fine-tune protein translation by promoting ribosome A-site binding. Proceedings of the National Academy of Sciences of the United States of America, 110(30), 12289-12294. doi:10.1073/pnas.1300781110.

Schauer, N., Steinhauser, D., Strelkov, S., Schomburg, D., Allison, G., Moritz, T., et al. (2005). GC-MS libraries for the rapid identification of metabolites in complex biological samples. FEBS Letters, 579(6), 1332-1337. doi:10.1016/j.febslet.2005.01. 029.

Sikorski, R. S., \& Hieter, P. (1989). A system of shuttle vectors and yeast host strains designed for efficient manipulation of DNA in Saccharomyces cerevisiae. Genetics, 122(1), 19-27.

Slupsky, C. M., Rankin, K. N., Wagner, J., Fu, H., Chang, D., Weljie, A. M., et al. (2007). Investigations of the effects of gender, diurnal variation, and age in human urinary metabolomic profiles. Analytical Chemistry, 79(18), 6995-7004. doi:10. 1021/ac0708588.

Stacklies, W., Redestig, H., Scholz, M., Walther, D., \& Selbig, J. (2007). pcaMethods-a bioconductor package providing PCA methods for incomplete data. Bioinformatics, 23(9), 1164-1167. doi:10.1093/bioinformatics/btm069.

Tigano, M., Ruotolo, R., Dallabona, C., Fontanesi, F., Barrientos, A., Donnini, C., et al. (2015). Elongator-dependent modification of cytoplasmic tRNALysUUU is required for mitochondrial function under stress conditions. Nucleic Acids Research, 43(17), 8368-8380. doi:10.1093/nar/gkv765.

Tükenmez, H., Xu, H., Esberg, A., \& Byström, A. S. (2015). The role of wobble uridine modifications in +1 translational frameshifting in eukaryotes. Nucleic Acids Research, 43(19), 9489-9499. doi:10.1093/nar/gkv832.

Venables, William N., \& Ripley, B. D. (2002). Modern applied statistics with $S$. New York: Springer.

Vendeix, F. A., Murphy, F. V. T., Cantara, W. A., Leszczynska, G., Gustilo, E. M., Sproat, B., et al. (2012). Human tRNA(Lys3)(UUU) is pre-structured by natural modifications for cognate and wobble codon binding through keto-enol tautomerism. Journal of Molecular Biology, 416(4), 467-485. doi:10.1016/j. jmb.2011.12.048.

Winkler, G. S., Kristjuhan, A., Erdjument-Bromage, H., Tempst, P., \& Svejstrup, J. Q. (2002). Elongator is a histone H3 and H4 acetyltransferase important for normal histone acetylation levels in vivo. Proceedings of the National Academy of Sciences of the United States of America, 99(6), 3517-3522.

Winkler, G. S., Petrakis, T. G., Ethelberg, S., Tokunaga, M., Erdjument-Bromage, H., Tempst, P., et al. (2001). RNA polymerase II elongator holoenzyme is composed of two discrete subcomplexes. Journal of Biological Chemistry, 276(35), 32743-32749.

Wittschieben, B. O., Otero, G., de Bizemont, T., Fellows, J., Erdjument-Bromage, H., Ohba, R., et al. (1999). A novel histone acetyltransferase is an integral subunit of elongating RNA polymerase II holoenzyme. Molecular Cell, 4(1), 123-128.

Zinshteyn, B., \& Gilbert, W. V. (2013). Loss of a conserved tRNA anticodon modification perturbs cellular signaling. PLoS Genetics, 9(8), e1003675. doi:10.1371/journal.pgen.1003675. 